\title{
Acute pancreatitis as the first manifestation of duodenal MALT lymphoma
}

\author{
Ivona Simkova ${ }^{a}$, Karel Urbanek ${ }^{b}$, Vlastimil Prochazka ${ }^{a}$, Michal Konecny ${ }^{a}$, Jan Gregara, Marie Geierova ${ }^{c}$, Vit Prochazkad, \\ Eva Buriankova ${ }^{e}$
}

Background. Possibly any tumor that can cause mechanical obstruction of the distal bile duct can induce acute pancreatitis. However, acute pancreatitis as the first clinical manifestation of duodenal lymphoma is extremely rare.

Objective. To report the case of a patient with acute pancreatitis as an extremely rare first manifestation of duodenal MALT lymphoma and possible association with erythema nodosum.

Methods. Case report of a 66-year-old woman who was diagnosed with acute pancreatitis caused by infiltration with duodenal lymphoma.

Results. Acute pancreatitis was confirmed by CT imaging. Detailed investigation revealed a duodenal mass causing pancreatic injury. Histological analysis established the diagnosis of MALT lymphoma. The patient's medical history also included erythema nodosum. Complete remission of the malignancy was achieved with chemotherapy.

Conclusion. This is the first published case report of acute pancreatitis caused by the growth of duodenal MALT lymphoma. An association with erythema nodosum is possible.

Key words: erythema nodosum, extranodal marginal zone lymphoma, pancreatic inflammation

Received: March 29, 2015; Accepted with revision: September 10, 2015; Available online: October 22, 2015 http://dx.doi.org/10.5507/bp.2015.047

${ }^{a}$ Department of Internal Medicine II - Gastroenterology and Hepatology, Faculty of Medicine and Dentistry, Palacky University Olomouc and University Hospital Olomouc, Czech Republic

${ }^{b}$ Department of Pharmacology, Faculty of Medicine and Dentistry, Palacky University Olomouc and University Hospital Olomouc 'Department of Clinical and Molecular Pathology, Faculty of Medicine and Dentistry, Palacky University Olomouc and University Hospital Olomouc

${ }^{d}$ Department of Hemato-Oncology, Faculty of Medicine and Dentistry, Palacky University Olomouc and University Hospital Olomouc ${ }^{e}$ Department of Nuclear Medicine, Faculty of Medicine and Dentistry, Palacky University Olomouc and University Hospital Olomouc Corresponding author:Vlastimil Prochazka, e-mail: Vlastimil.Prochazka@post.cz

\section{INTRODUCTION}

The current World Health Organization classification of tumors of hematopoietic and lymphoid tissues, updated in 2008, describes three types of marginal zone lymphoma (MZL) - extranodal, nodal and splenic ${ }^{1}$.

Extranodal marginal zone B-cell lymphoma, also known as mucosa-associated lymphoid tissue lymphoma (MALT lymphoma), comprises 7-8\% of all B-cell lymphomas and accounts for approximately $70 \%$ of all MZLs. MALT lymphomas constitute about $50 \%$ of gastric lymphomas. Conversely, about $50 \%$ of them are found in the digestive tract and $34 \%$ in the stomach. However, they can develop in various organs such as the salivary gland, thyroid, lung and breast. They may arise in organs both normally containing and lacking lymphoid tissue. They most commonly occur between the ages of 50 and 60 and a slight female predominance is noticeable $e^{2-4}$.

MALT lymphomas arise from postgerminal center (marginal zone) B cells (centrocyte-like cells). Tumorous tissue predominantly consists of the aforementioned neoplastic small marginal zone B lymphocytes. Monocytoid elements, scattered immunoblasts, centroblast-like cells and various numbers of plasma differentiated cells can be present. The infiltrates are visible in the marginal zone of reactive lymphoid B-cell follicles which expand in the interfollicular regions. The neoplastic cells typically infiltrate the epithelium forming lymphoepithelial lesions (LEL) in epithelial organs. LEL identification is considered the gold standard diagnostic test ${ }^{3-6}$.

Various localizations of marginal lymphomas have been attributed to specific lymphocyte homing, i.e. regulated trafficking of lymphocytes in the tissues: the lymph nodes, the spleen or mucosa-associated lymphoid tissue. A chronic inflammatory disorder caused by infection, autoimmunity or another unknown stimulus can result in accumulation of extranodal lymphoid tissue. Secondary acquired MALT in various sites may form the substrate for lymphoma development ${ }^{6}$.

Gastric MALT lymphoma is strongly associated with Helicobacter pylori infection. H. pylori eradication is a well-established initial treatment modality in $\mathrm{H}$. pyloripositive patients diagnosed with mucosal or submucosal gastric MALT-lymphoma. Other suspected infectious organisms that may lead to accumulation of mucosa-associated lymphoid tissue preceding MALT lymphoma include Chlamydia psittaci (ocular adnexal MALT lymphoma), Campylobacter jejuni (immunoproliferative small inestinal disease), Borrelia burgdorferi (cutaneous MALT lymphoma). However, there is a great variability in the power of these associations $s^{6,7}$. 
The role of hepatitis $\mathrm{C}$ virus ( $\mathrm{HCV}$ ) infection in the pathogenesis of MALT lymphoma is still under discussion. The relation between this hepatotropic and lymphotropic virus and mixed cryoglobulinemia, a benign lymphoproliferative disorder, is well known. However, its contribution to the pathogenesis of lymphomas is not perfectly clear. It has been discussed as a potential infectious co-factor in the multiple-stage pathogenesis of MALT lymphomas ${ }^{8}$.

Both Hashimoto's thyroiditis and Sjögren's syndrome represent autoimmune disorders connected with thyroid and salivary lymphomas. Patients with Sjögren's syndrome or lymphoepithelial sialadenitis have a 44-fold increased risk of developing overt lymphoma. MALT lymphoma has been diagnosed in the majority of these cases $(85 \%)$. Evidence of previous thyroiditis has been found in not less than $94 \%$ of thyroid lymphomas ${ }^{6}$.

Although the immunophenotype of MALT lymphomas is not entirely specific, malignant cells express a characteristic immunophenotype of B lymphocytes. Immunostaining can prove pan B cell markers CD 19, CD 20, CD 22 and membrane immunoglobulins, excluding IgD. Light chain restriction is usually detected. In most cases, CD 5, CD 10 and CD 23 markers are found negative. MALT lymphomas are found to be cyclin D1negative as well ${ }^{6,9,10-13}$. Cytogenetic heterogeneity of tumors is also observed. Trisomy of chromosome 3 is detected most frequently. Chromosomal translocation $\mathrm{t}(11 ; 18)$ (q21; q22) is found in $30-40 \%$ of MALT lymphomas ${ }^{13-18}$.

Duodenal MALT lymphoma represents a very rare localization of this neoplasm. Xiang et al. found only 17 previous cases in the English literature in 2004. If the reported cases listed below are summarized, symptomatology of this entity can vary from patient to patient. It can present as upper gastrointestinal bleeding, recurrent vomiting, abdominal distension, gastric outlet obstruction or general symptoms of malignancy, such as weakness or weight $\operatorname{loss}^{5,19}$.

Contrary to gastric MALT lymphoma, the role of Helicobacter pylori remains unclear. Nagashima et al. reported a regression of duodenal MALT lymphoma following eradication ${ }^{20,21}$.

Bile duct obstruction can cause acute pancreatitis as well as a pathological process in the duodenum, or rather in the region of the papilla of Vater. The most common tumors considered in the differential diagnosis of bile duct obstruction are cholangiocellular carcinoma and pancreatic adenocarcinoma. Ampullary adenocarcinoma represents a relatively rare malignancy; however, it typically manifests with painless obstructive jaundice. A retrospective study of 35 patients with lymphoma and obstructive jaundice was published in 2010. Large B cell lymphomas were found in the majority of cases ${ }^{22}$. Hypothetically, any tumor that can cause mechanical obstruction of the distal bile duct or the ampulla of Vater can induce acute pancreatitis.

Selden et al. describe a rare case of a 56-year-old female diagnosed with duodenal MALT lymphoma manifested by upper gastrointestinal bleeding and acute pancreatitis. It has been the first abstract concerning pancreatitis caused by the growth of a duodenal MALT lymphoma ${ }^{23}$. In our case report, we describe another case of acute pancreatitis caused by duodenal MALT lymphoma.

\section{CASE REPORT}

A 66-year-old woman was admitted to our hospital with symptoms of acute biliary pancreatitis. The patient underwent cholecystectomy 22 years ago. The patient suffered from epigastric pain which had been gradually intensifying for 14 days and peaked 24 hours before admission. A detailed history revealed sings of cholestasis within the two preceding weeks. The patient complained of pruritus, acholic stools and dark urine. Physical examination was within normal limits, except for epigastric pain on palpation.

Laboratory analysis supported the clinical suspicion of acute pancreatitis. Serum levels of amylase and lipase were significantly increased. The level of alpha-amylase exceeded 22 microkatal ( $\mu \mathrm{kat} / \mathrm{L}$ ) (normal range 0.47-1.67 $\mu \mathrm{kat} / \mathrm{L}$ ); lipase was elevated to $55 \mu \mathrm{kat} / \mathrm{L}$ (normal range 0.22-1.2 $\mu \mathrm{kat} / \mathrm{L}$ ). Furthermore, elevations of alkaline phosphatase (ALP) and gamma-glutamyl transferase (GGT) indicated the possibility of bile duct obstruction. ALP was above $8 \mu \mathrm{kat} / \mathrm{L}$ (normal range 0.62-2.4 $\mu \mathrm{kat} / \mathrm{L}$ ), GGT 11 $\mu \mathrm{kat} / \mathrm{L}$ (normal range 0.0-0.6 $\mu \mathrm{kat} / \mathrm{L}$ ). Abdominal ultrasound imaging was performed and revealed dilatation of both the pancreatic duct ( 4 millimeters) and intrahepatic biliary ducts (Fig. 1).

Based on the aforementioned findings, endoscopic retrograde cholangiopancreatography (ERCP) was performed (endoscope Olympus EXERA TJF 160VR). Neither drainage nor cholangiography of the bile ducts were feasible because of exophytic ulcerating infiltration in the region of the papilla of Vater. Although the expected benefit of ERCP was not achieved, tissue samples from the duodenal mass could be collected by the biopsy forceps. Clinical course, medical history, and laboratory testing led to suspicion of acute biliary pancreatitis. ERCP was available in contrast with magnetic resonance cholangiopancreatography (MRCP).

Due to a suspected malignant process in the pancreas

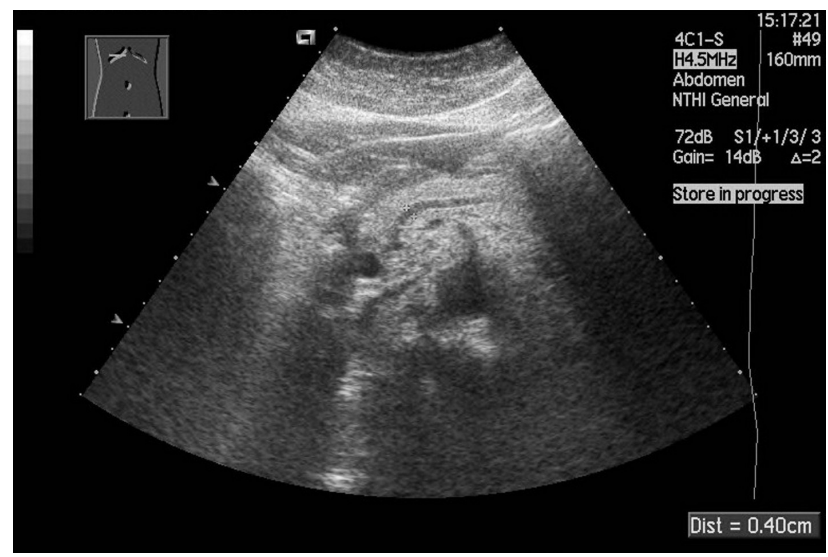

Fig. 1. Abdominal ultrasound imaging: pancreas with 4 millimeter pancreatic duct dilatation. 
or ampulloma, abdominal computed tomography scanning was performed as a further diagnostic procedure. It confirmed acute pancreatitis (Balthazar B, CTSI 1). The pancreatic duct was clearly visible; however, direct signs of a malignant process were not found. Gallstones were not detected on CT scans, which was performed shortly after ERCP. Duodenal thickening was visible on these initial CT scans.

Gastrointestinal X-ray contrast imaging was performed with the intention to verify possible duodenal obstruction, however patient had no clear signs of gastrointestinal obstruction. Neoplasm formed tight stenosis of the duodenum, but we excluded gastric outflow obstruction. Examination showed a 4 centimeters long stenosis within the D2 segment of the duodenum which was permeable to the contrast agent (Fig. 2).

A malignancy was highly likely, despite the fact that the first tissue sample failed to confirm the presence of any cancer tissue. Exacerbated chronic inflammation was described. Upper gastrointestinal endoscopy (endoscope Olympus EXERA II GIF Q 180ISBI) was carried out in order to collect more biopsy samples for histological analysis. Unexpectedly, histological and immunohistochemical testing confirmed mucosa-associated lymphoid tissue (MALT) lymphoma. Other forms of B-cell lymphomas were differentiated due to morphological and immunohistochemical analysis. Hematoxilin-eosin staining clearly demonstrated the morphology of lymphoma cells (Fig. 3A). Ki67 index was carried out to assess the proliferative fraction, that was less than $15 \%$ (Fig. 3B). CD20 posi-

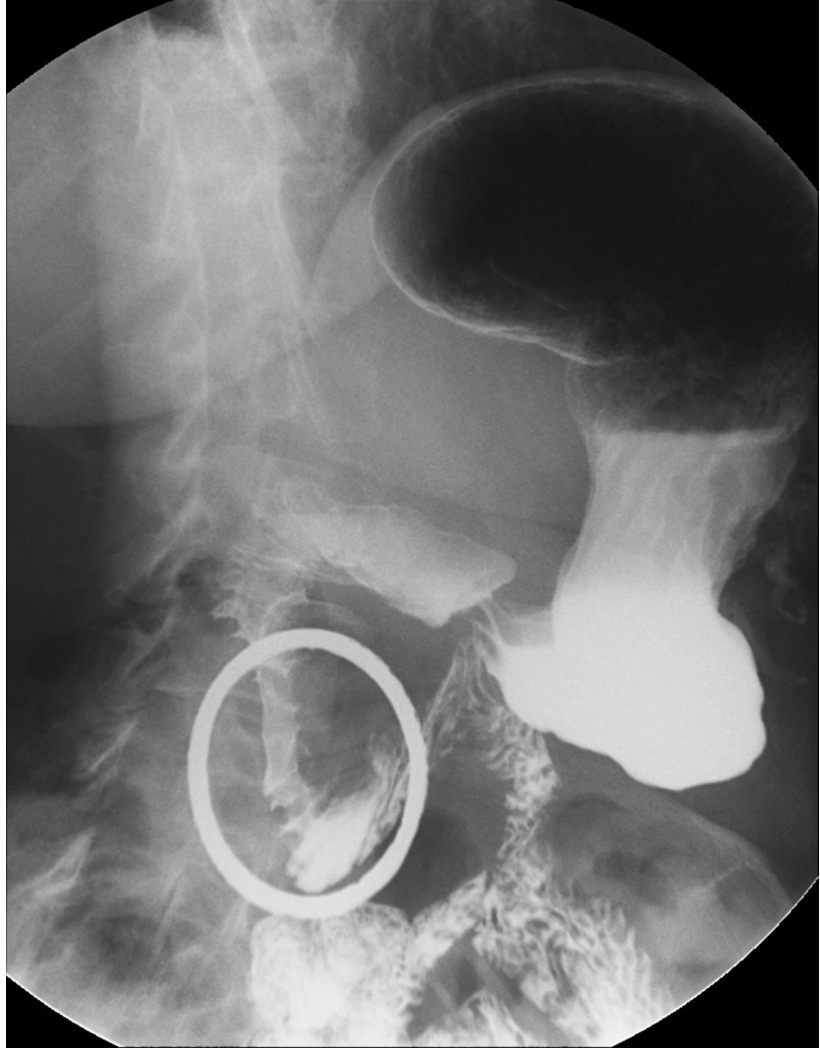

Fig. 2. Gastrointestinal X-ray contrast imaging: 4 centimeters long stenosis of D2 duodenum pervious for contrast agent.

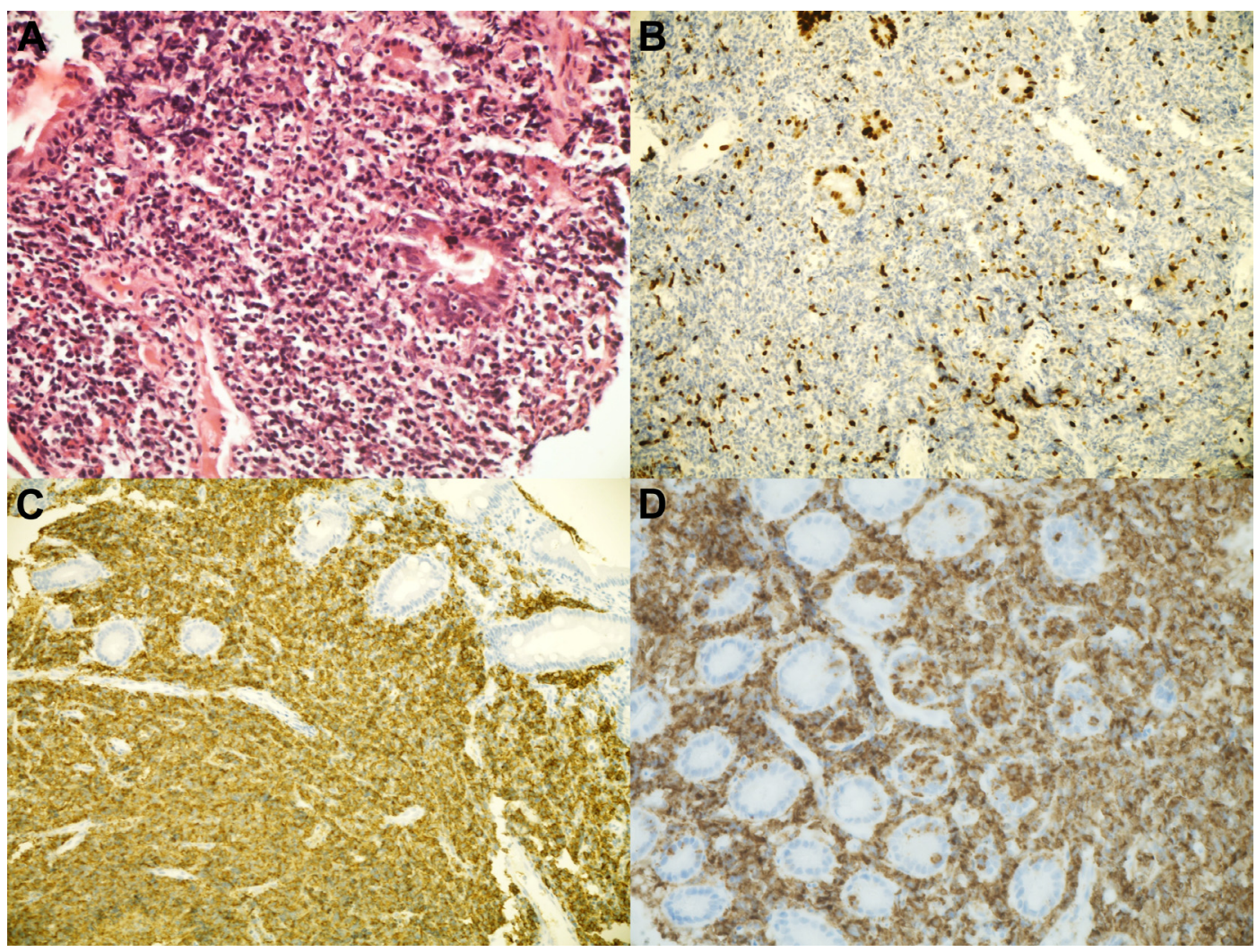

Fig. 3A. Hematoxillin-eosin staining 3B. Ki 67 stain 3C. difuse infiltrate of CD 20-positive B lymphoid cells (margine zone cells) 3D. Lymphoepithelial lesions. 
tive cell diffuse infiltration was detected by immunohistochemistry (Fig 3C). CD3, CD5, CD10, CD23, CD 43 marekers and cyclin D1 were negative. Diffuse growth and CD 10 negativity was observed in our case. It allowed to distinguished MALT lymphoma from follicular lymphoma grade 1. Lymphoepithelial lesions were clearly visible (Fig 3D). This typical sign of MALT lymphoma supported the final diagnosis. Other types of lymphomas were excluded by morphology, immunohistochemistry and by assessing of Ki67 proliferation index.

Fluorescence in situ hybridization (FISH) examination was completed later. The material, which is routinely stored in the Department of Clinical and Molecular Pathology, was used. Probe hybridization was succesful, therefore the result is valid, as was confirmed by hematologist and cytogeneticist. MALT 1 gene translocation has not been proved, however 3 copies of MALT 1 gene has been described. MALT 1 is located in chromosome 18 , therefore this finding parallels chromosome 18 trisomy. Trisomy 18 is a non-specific but also not infrequent finding in MALT lymphomas. The frequencies in which the translocation or trisomies occur vary markedly with the primary site of the disease. Duodenal site has not been studied yet. Transformation of immunoglobulin heavy chain / IgH/ gene, located in chromosome 14, which is translocation partner in the case of follicular lymphoma in the $\mathrm{t}(14 ; 18)$ or in the case of mantle cell lymphoma in the $\mathrm{t}(11 ; 14)$, was not present. Therefore these diagnoses are highly improbable. In conclusion, diagnosis of MALT lymphoma has been supported by these findings. Histopathology findings are precise, immunohistochemistry provides additional information.

Staging of the disease was performed by using Positron Emission Tomography/Computed Tomography Scanning (PET/CT, equipment Siemens Biograph 16) which revealed accumulation of fluorodeoxyglucose in the duodenum, the hepatic hilar lymph node and a single mesenteric lymph node (Fig. 4). We performed PET/CT at the time, when no clinical, laboratory and CT signs of pancreatitis were presented. The International Prognostic Index (IPI) was determined to be 3 points, with 1 point for each poor prognostic factor: age over 60, stage IV A of the lymphoma, and serum LDH above $3.8 \mu \mathrm{kat} / \mathrm{L}$ (normal range 2.25-3.55 $\mu \mathrm{kat} / \mathrm{L})$.

The patient underwent standard therapy for acute pancreatitis, which included fluid administration, nutritional

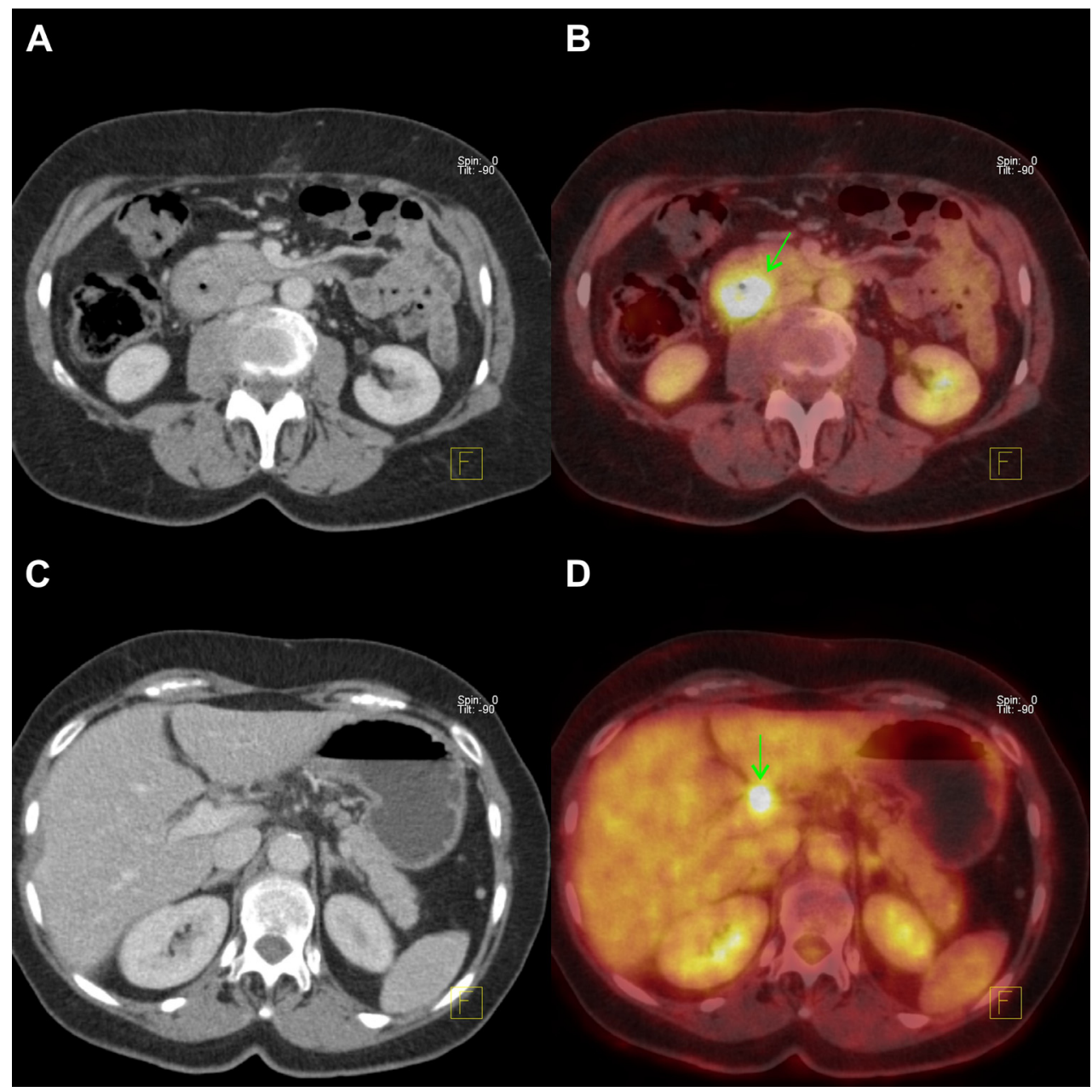

Fig. 4A. CT scan - circular duodenum wall thickening 4B. PET/CT fusion - FDG uptake in the duodenum wall 4C. CT scan - enlarged liver hilum lymph node 4D. PET/CT fusion - FDG uptake in liver hilum lymph node. 
support, analgesia, parenteral proton pump inhibitors and prevention of thromboembolic complications. Monitoring of the patient was conducted at an intensive care unit for 4 days, and then continued at a standard department. Clinical symptoms and laboratory findings normalized within 11 days without a need for invasive biliary intervention.

The patient could be discharged from hospital care and referred to the Department of Hemato-Oncology. A detailed investigation was finalized, including immunophenotyping of peripheral blood and bone marrow lymphocytes and molecular biology tests. The patient underwent sternal bone marrow aspiration and trephine biopsy of the iliac bone. Bone marrow examination excluded tumor infiltration. Microbiological screening was also performed. Among other things, tests for hepatitis B, hepatitis C, EBV, CMV infection and Helicobacter pylori were negative.

Systemic chemotherapy could then be started. Complete remission of the tumor with negative PET/CT (Fig. 5), upper endoscopy and negative duodenal biopsy was achieved by using R-CHOP combination immunochemotherapy (rituximab, cyclophosphamide, doxorubi- cin, vincristine and prednisone), i.e. 8 months after the diagnosis. Patient underwent 6 cycles of R-CHOP chemotherapy. The patient has now been free of symptoms for 3 years.

\section{DISCUSSION}

Extranodal lymphomas are particularly manifested by local symptoms caused by organ infiltration. Symptoms of gastrointestinal lymphomas may vary from dyspepsia, abdominal pain, nausea, vomiting, diarrhea, anemia, weight loss, and malabsorption to the signs of gastrointestinal obstruction, ulceration, intestinal perforation or intussusception. Gastrointestinal bleeding has also been reported. Conversely, a few patients were reportedly asymptomatic ${ }^{24}$.

Duodenal MALT lymphoma is a very rare entity. Symptoms are non-specific. Clinical manifestation depends on the exact location of the tumor, the rapidity of progression of the disease and whether the obstruction is caused by the tumorous mass. Xiang et al. reported a case of a 64-year-old woman with upper gastrointestinal

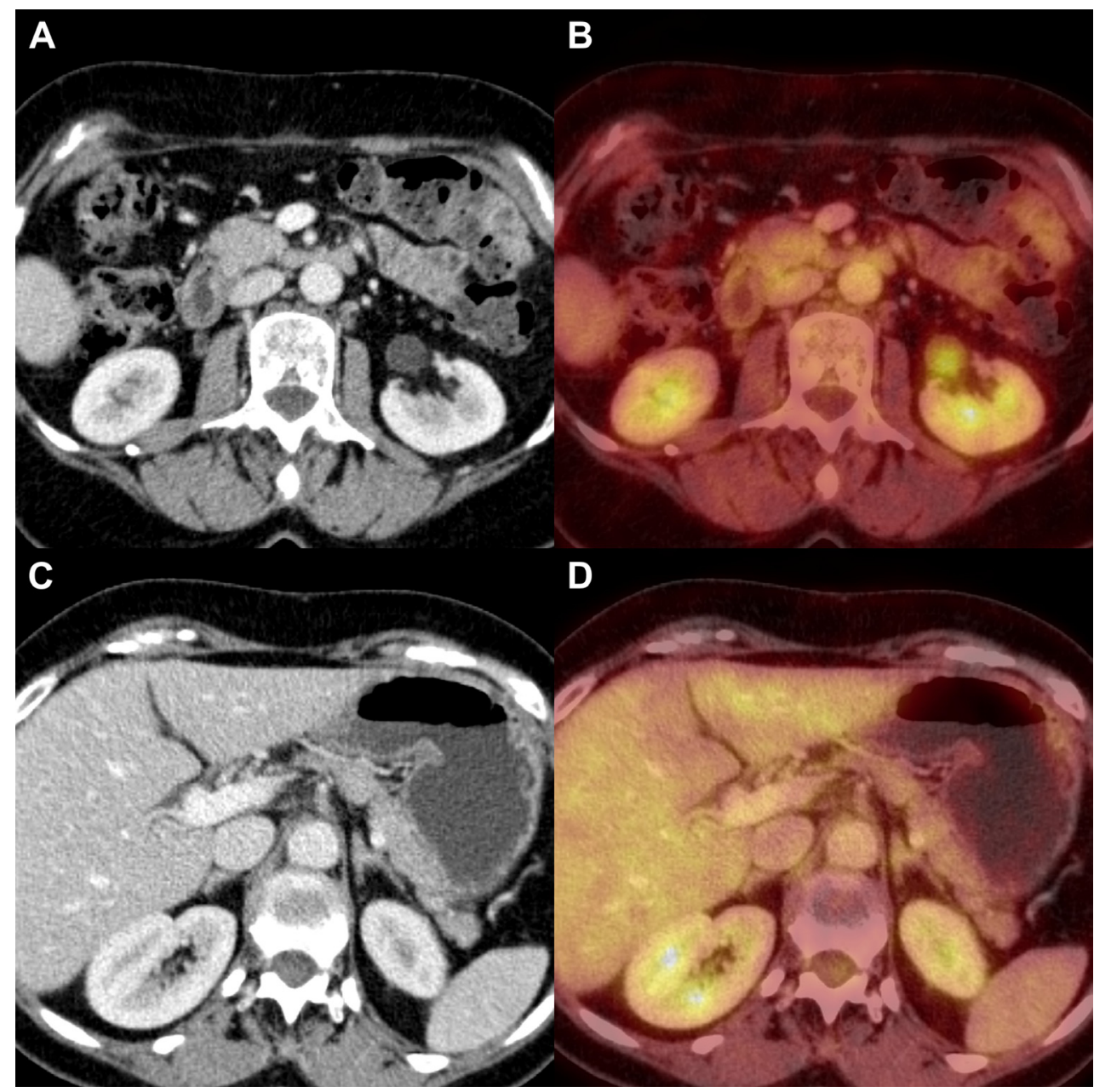

Fig. 5A. CT scan after treatment - normal duodenum wall thickness 5B. PET/CT fusion after treatment - normal FDG uptake in the duodenum wall 5C. CT scan after treatment - no obvious enlargement of liver hilum lymph node 5D. PET/CT fusion after treatment - no FDG uptake in liver hilum lymph node. 
bleeding manifested by melena. Gastroscopy revealed an ulcerating lesion in the duodenum. Histological testing confirmed low-grade MALT lymphoma ${ }^{19}$.

Ndzengue et al. detailed a case of a 73-year-old woman who had a 2-week history of general weakness, recurrent projectile vomiting containing food particles, abdominal distension, discomfort and significant weight loss. Abdominal and pelvic CT scan showed gastric distension suggestive of gastric outlet obstruction. However, no pancreatic or duodenal tumorous mass was detected. Upper endoscopy revealed a tumorous mass obturating the distal part of the duodenum. Histological investigation confirmed this tumor to be marginal B cell lymphoma 5 .

Another interesting case of duodenal MALT lymphoma diagnosed together with multiple intra-abdominal thromboses and hepatitis $\mathrm{C}$ virus infection was published in 2009 by Ozkok et al. ${ }^{8}$.

Our case report is rare not only for the duodenal type of MALT lymphoma, but also for the unique manifestation of this neoplasm. The patient was first diagnosed with acute pancreatitis and only a further detailed investigation revealed the rare cause of this acute condition. A malignant process incipient in the pancreas or ampulloma had been considered in the differential diagnosis of duodenal tumor until histological analysis elucidated its origin. It played a fundamental role in the approach to the patient and subsequent treatment.

The patient underwent a series of tests, but no underlying infection or autoimmune disease was proved at the time of malignant lymphoma diagnosis. A summary of the patient's medical history also included erythema nodosum diagnosed in 2004; therefore, sarcoidosis was excluded repeatedly. The patient had been followed by a pulmonologist and a rheumatologist continuously; nevertheless, no pathology related to erythema nodosum had been found. A previous chlamydia infection was detected; however, the condition was finally considered as an idiopathic form of the disease.

Medical history of erythema nodosum may be interesting from several viewpoints. Association of this skin inflammatory reaction with non-Hodgkin's lymphoma has been mentioned in the literature ${ }^{25}$. Protracted and recurrent course of the skin lesions could be related to latent malignancy, although the patient had no signs of erythema nodosum at the time of diagnosis. Another important question concerns the possibility of an underlying infection. Despite the fact that the patient underwent a series of tests to find a possible infection causing skin manifestation with negative results, a latent or spontaneously resolved infection could still have been present. The same hypothetic infectious agent could have contributed to the pathogenesis of MALT lymphoma.

Another fact discovered in the patient's medical history was the isolated elevation of gamma-glutamyl transferase (GGT), first documented in 2005. Laboratory tests repeatedly confirmed mild anicteric cholestasis as well as slight alanine aminotransferase elevation in the further course. Consequently, ERCP was suggested, but it was eventually refused at that time. Repeated abdominal ultrasound imaging failed to find any pathology of the bile ducts. The only pathological finding were ultrasound signs of hepatic steatosis, later confirmed by histological analysis of liver biopsy specimens.

\section{CONCLUSION}

In conclusion, this case report aims to highlight the possibility of a rare presentation of this uncommon neoplasm. Whether our patient's erythema nodosum and her previous episodes of anicteric cholestasis were associated with MALT lymphoma, or it was just a coincidence, remains an open question.

Author contributions: IS: manuscript writing, first author; $\mathrm{KU}$ : literature search; VP: revision of some parts of the text, literature search, CT scan descriptions ; MK: description of the endoscopy procedures; JG: description of the endoscopy procedures; MG: description of the histopathology findings; VP: description of the FISH method; EB: description of the PET/CT scan.

Conflict of interest statement: The authors state that there are no conflicts of interest regarding the publication of this article.

\section{REFERENCES}

1. Campo E, Swerdlow SH, Harris NL, Pileri S, Stein H, Jaffe ES.The 2008 WHO classification of lymphoid neoplasms and beyond: evolving concepts and practical applications. Blood 2011;117(19):5019-32.

2. Mazloom A, Medeiros LJ, McLaughlin PW, Reed V, Cabanillas FF, Fayad LE, Pro B, Gonzalez G, Iyengar P, Urbauer DL, Dabaja BS. Marginal Zone Lymphomas. Factors that affect the final outcome. Cancer 2010;116(18):4291-8

3. Tomizawa Y, Seki M, Mori M. Unusual presentation of localized gastric mucosa-associated lymphoid tissue lymphoma mimicking poorly differentiated gastric adenocarcinoma. Case Rep Gastroenterol 2012;6(1):47-51.

4. Park H, Chung JW, Kim AJ, Park SY, Rim MY, Jang YR, Lee JH, Park S. A Case of Rectal Mucosa-associated Lymphoid Tissue Lymphoma Diagnosed by Endoscopic Unroofing Technique. Korean J Gastroenterol 2012;59(6):428-32.

5. Ndzengue A, Khurana R, Mora M, Rafal RB, Trauber D, Mansour M, Posner GL, Jaffe EA. Gastric marginal zone B cell lymphoma of the duodenum. Case Rep Gastroenterol 2011;5(3):578-82.

6. Isaacson PG, Chott A, Nakamura S, Muller-Hermelink HK, Harris NL, Swerdlow SH. Extranodal marginal zone lymphoma of mucosa-associated lymphoid tissue (MALT lymphoma) In: Swerdlow SH, Campo E, Harris NL, Jaffe ES, Pileri SA, Stein H, editors. WHO classification of tumours of haematopoietic and lymphoid tissues. Lyon: IARC; 2008. pp. 214-7.

7. Song KH, Yun M, Kim JH, Yang WI, Kang DR, Chung JB, Lee YC. Role of 18F-FDG PET Scans in Patients with Helicobacter pylori -Infected Gastric Low-Grade MALT Lymphoma. Gut Liver 2011;5(3):308-14.

8. Ozkok A, Tufan F, Namli S, Bulakci M, Pinarbasi B, Dogan O, Karan MA, Tascioglu C. Mucosa-associated lymphoid tissue lymphoma of the duodenum together with multiple intra-abdominal thromboses and hepatitis C virus infection: a case report. Cases J 2009;2:9354.

9. Arends JE, Bot FJ, Gisbertz IAM, Schouen HC. Expression of CD 10, CD 75 and CD 43 in MALT lymphoma and their usefulness in discriminating MALT lymphoma from follicular lymphoma and chronic gastritis. Histopathology 1999;35:209-15.

10. Ferry JA, Yang W, Zukerberg LR, Wotherspoon AC, Arnold A, Harris NL. CD 5+ extranodal marginal zone B-cell (MALT) lymphoma. Amer J Clin Pathol 1996;105:31-7. 
11. Thieblemont C, Bastion Y, Berger F, Rieux C, Salles G, Dumontet Ch, Felman P, Coiffier B. Mucosa-associted lymphoid tissue gastrointestinal and nongastrointestinal lymphoma behavior: Analysis of 108 patients. J Clin Oncol 1997;15:1624-30.

12. Vose JM, Chair S, Fisher RI, Speaker S, Lister AA: Unusual lymphoma entities: Evaluation and management. Amer Soc Clin Oncol 1997.

13. Schechter NR, Yahalom J. Low grade MALT Iymphoma of the stomach: A review of treatment options. Int J Rad Oncol Biol Phys 2000;46:1093-103.

14. Auer IA, Gascoyne RD, Connors JM, Cotter FE, Greiner TC, Sanger WG Horsman DE. $t(11 ; 18)(q 21 ; q 21)$ is the most common translocation in MALT lymphomas. Ann Oncol 1997;8:979-85.

15. Chott A, Raderer M. New developments in extracutaneous lymphomas. Semin Cutaneous Med Surg 2000;19:149-56.

16. Morgner A, Bayerdorffer E, Neubauer A, and Stolte M. Gastric MALT lymphoma and its relationship to Helicobacter pylori infection: Management and pathogenesis of the disease. Micr Res Technique 2000;48:349-56.

17. Nakamura T, Nakamura S, Yonezumi M, Suzuki T, Matsuura A, Yatabe $Y$ Yokoi T, Ohashi K, Seto M. Helicobacter pylori and the $t(11 ; 18)(q 21 ; q 21)$ translocation in gastric low-grade B-cell lymphoma of mucosa-associated lymphoid tissue type. Jpn J Cancer Res 2000;91:301-9.

18. Ott G, Katzenberger T, Greiner A, Kalla J, Rosenwald A, Heinrich U Ott MM, Müller-Hermelink HK. The t(II;18)(q21; q21) chromosome translocation is a frequent and specific aberration in low-grade but not high-grade malignant non-Hodgkin's lymphomas of the mucosa associated lymphoid tissue (MALT-) type. Cancer Res 1997;57:3944-8.
19. Xiang Z, Onoda N, Ohira M, Yukawa T, Uchida T, Arakawa T, Wakasa $\mathrm{K}$, Chung KH. Mucosa-associated lymphoid tissue lymphoma of the duodenum: a report of a case resistant to Helicobacter pylori eradication. Hepatogastroenterology 2004;51(57):732-5.

20. Peng AP, Chen MK, Shen L, Luo HS, Chen YH. Simultaneous primary low-grade mucosa-associated lymphoid tissue lymphoma of stomach and duodenum. Digestion 2010;81(2):130-3.

21. Nagashima R, Takeda H, Maeda K, Ohno S, Takahashi T. Regression of duodenal mucosa-associated lymphoid tissue lymphoma after eradication of Helicobacter pylori. Gastroenterology 1996;111:16748.

22. Ross WA, Egwim Cl, Wallace MJ, Wang M, Madoff DC, Lee JH Outcomes in lymphoma patients with obstructive jaundice: a cancer center experience. Dig Dis Sci 2010;55(11): 3271-7.

23. Selden M, Sanchez P, Saddedin E, Laya S, Clarkston W. MALT Lymphoma of the Duodenum: A Rare Cause for Upper Gastrointestinal Bleeding and Pancreatitis. (Abstr.) Am J Gastroenterol 2007;102:S302.

24. Bautista-Quach MA, Ake CD, Chen M, Wang J. Gastrointestinal lymphomas. Morphology, immunophenotype and molecular features. J Gastrointest Oncol 2012;3(3):209-25.

25. Thomson GT, Keystone EC, Sturgeon JF, Fornasier V. Erythema nodosum and non-Hodgkin's Iymphoma. J Rheumatol 1990;17(3):383-5. 